\title{
Theoretical study on dynamical planar-chirality switching in checkerboard-like metasurfaces
}

\author{
Yoshiro Urade ${ }^{1, *}$, Yosuke Nakata ${ }^{2}$, Toshihiro Nakanishi ${ }^{1}$, and Masao Kitano ${ }^{1, a}$ \\ 1 Department of Electronic Science and Engineering, Kyoto University, Kyoto 615-8510, Japan \\ 2 Center for Energy and Environmental Science, Shinshu University, 4-17-1 Wakasato, Nagano 380-8553, Japan
}

Received 10 September 2016 / Accepted 15 November 2016

\begin{abstract}
In this paper, we show that the handedness of a planar chiral checkerboard-like metasurface can be dynamically switched by modulating the local sheet impedance of the metasurface structure. We propose a metasurface design to realize the handedness switching and theoretically analyze its electromagnetic characteristic based on Babinet's principle. Numerical simulations of the proposed metasurface are performed to validate the theoretical analysis. It is demonstrated that the polarity of asymmetric transmission for circularly polarized waves, which is determined by the planar chirality of the metasurface, is inverted by switching the sheet impedance at the interconnection points of the checkerboard-like structure. The physical origin of the asymmetric transmission is also discussed in terms of the surface current and charge distributions on the metasurface.
\end{abstract}

Key words: Planar chirality, Asymmetric transmission, Tunable metamaterials, Babinet's principle.

\section{Introduction}

Chiral structures have been one of the most interesting subjects in metamaterials research $[1,2]$. In analogy to the chirality of three-dimensional structures, a planar structure is said to be planar chiral if it has no line of mirror symmetry in its structure plane (e.g., an Archimedean spiral) [3, 4]. In other words, a planar chiral structure cannot be superimposed on its in-plane mirror image by in-plane rotations and/ or translations. Note that the handedness of a planar chiral structure is reversed if it is observed from the opposite side of its structure plane. Polarization effects related to the planar chiral structures for electromagnetic waves have been studied in terms of optical activity and reciprocity for scattered light [5-7]. In addition, it has been identified that the total transmission intensity of circularly polarized waves normally incident on planar chiral structures depends on the direction of the wave propagation, provided that the planar chiral structures are anisotropic and lossy [8]. This phenomenon is called asymmetric transmission for circularly polarized waves and is consistent with the Lorentz reciprocity theorem, although it is seemingly nonreciprocal. Asymmetric transmission of planar chiral metamaterials has been experimentally reported in from microwave [8, 9] and terahertz [10] to optical frequency regions [11]. Moreover, it has also been extended to linearly polarized waves [12].

\footnotetext{
*e-mail: urade@giga.kuee.kyoto-u.ac.jp

a ORCID: 0000-0003-1065-8028
}

Recently, metamaterials and metasurfaces with dynamically tunable electromagnetic properties have been extensively studied towards applications to the advanced control of electromagnetic wave propagation [13, 14]. The recent progress has enabled the dynamical tuning of optical activity resulting from the chirality of artificial structures by employing photoexcitation of semiconductors [15-19], microelectro-mechanical systems $[20,21]$, and phase change materials [22]. On the other hand, the possibility of dynamical tuning of planar chirality and the associated asymmetric transmission for circular polarizations remains unexplored in literature.

In this paper, in order to realize dynamical planar-chirality switching, we utilize the singular characteristic of metallic checkerboard-like structures, which is critically dependent on the electrical connectivity at the interconnection points of their metallic patches [23-32]. We have shown that their characteristics can be understood in terms of Babinet's principle, which is extended to screens with finite sheet impedances [30, 31]. According to Babinet's principle, by switching the local sheet impedance at the interconnection points, the responses of the checkerboard-like structures can dynamically alternate between two contrasting behaviors, such as capacitive-inductive responses [33] and anisotropic transmission characteristics for orthogonal linear polarizations [34]. Here, we apply such dynamical-checkerboard designs to dynamical planar-chirality switching. In contrast to the previous designs [33, 34], a new design contains resonant planar chiral structures, which enable planar-chirality switching of the whole structure. 
This paper is organized as follows. First, in Section 2, we present the design of a metasurface that enables dynamical planar-chirality switching, and theoretically demonstrate its capability of planar-chirality switching based on Babinet's principle. In Section 3, we show the results of numerical simulations of the presented metasurface using the finite element method and discuss the physical origin of planarchirality switching. Finally, the conclusions are drawn in Section 4.

\section{Design and operation principle}

\subsection{Babinet's principle for the incidence of circularly polarized waves}

Before proceeding to a theoretical analysis of planar chiral metasurfaces based on Babinet's principle, we review Babinet's principle in particular for the incidence of circular polarization. Babinet's principle relates scattering problems of metasurfaces to those of their complementary structures. We assume that a metasurface in a vacuum has a spatially varying sheet impedance of $Z_{\mathrm{s}}(x, y)$, where $(x, y)$ is the coordinate on the metasurface. Then, the sheet impedance of its complementary metasurface is given by

$$
Z_{\mathrm{s}}^{(\mathfrak{c})}(x, y)=\frac{Z_{0}^{2}}{4 Z_{\mathrm{s}}(x, y)}
$$

where $Z_{0}$ is the impedance of a vacuum. This transformation is called the impedance inversion [30]. The electromagnetic fields in the two problems are related to each other if the incident fields in the complementary scattering problem satisfy $\left(\boldsymbol{E}_{\text {in }}^{(\mathrm{c})}, \boldsymbol{H}_{\text {in }}^{(\mathrm{c})}\right)=\left( \pm Z_{0} \boldsymbol{H}_{\text {in }}, \mp \boldsymbol{E}_{\text {in }} / Z_{0}\right)$, where $\left(\boldsymbol{E}_{\text {in }}, \boldsymbol{H}_{\text {in }}\right)$ is the incident electromagnetic field in the original problem. In particular, for the incidence of linearly polarized plane waves, this interchange of $\boldsymbol{E}$ and $\boldsymbol{H}$ is equivalent to the polarization rotation by $\pi / 2$. On the other hand, for the incidence of circularly polarized plane waves, the incident polarization state in the complementary problem is equal to that in the original problem, up to a phase factor of $\exp ( \pm \mathrm{i} \pi / 2)$, which depends on the handedness of the incident circular polarization [30]. For example, let us consider a problem shown in Figure 1a. Its complementary problem is depicted in Figure 1b. For the incidence of left circularly polarized (LCP) plane waves, we can derive the following equations via Babinet's principle [30]:

$$
\begin{aligned}
& \tilde{t}_{\mathrm{LL}}+\tilde{t}_{\mathrm{LL}}^{(\mathrm{c})}=1, \\
& \tilde{t}_{\mathrm{RL}}-\tilde{t}_{\mathrm{RL}}^{(\mathrm{c})}=0,
\end{aligned}
$$

where $\tilde{t}_{i j}$ and $\tilde{t}_{i j}^{(\mathrm{c})}(i, j=\mathrm{L}, \mathrm{R})$ denote the complex amplitude transmission coefficients from incident $j$ circular polarization to $i$ circular polarization in the original and complementary problems, respectively. For the incidence of right circularly polarized (RCP) waves, L and R in equations (2) and (3) are interchanged.

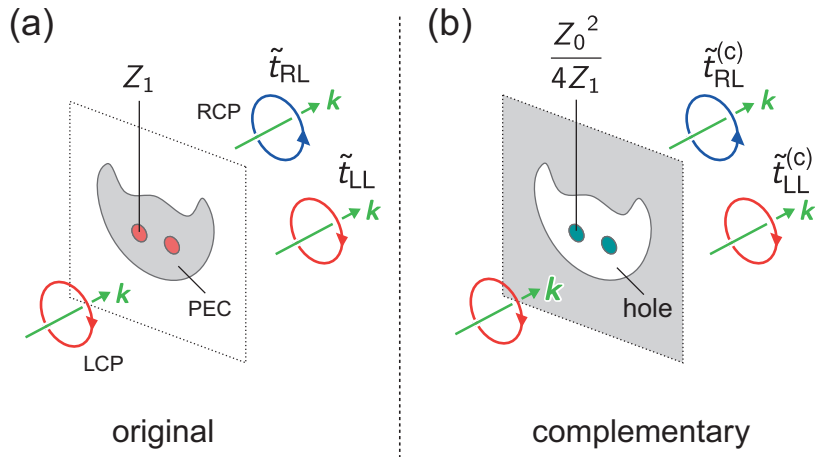

Figure 1. Illustration of Babinet's principle for the incidence of LCP plane waves. (a) Original problem; (b) complementary problem. The symbol $\boldsymbol{k}$ denotes the wavevector of the plane waves. This figure is based on Figure 2 of reference [31].

\subsection{Design of the metasurface for planar chirality switching}

Let us consider a planar chiral checkerboard-like metasurface in a vacuum, as shown in Figure 2a. We assume it to be infinitely periodic and infinitely thin. At the interconnection points of the perfect-electric-conductor (PEC) patches, there are variable impedance sheets with a sheet impedance of $Z_{\mathrm{s}}$. Deformed gammadions (hereafter, $\mathrm{R} \Gamma \mathrm{s}$ ) $[35,36]$ and their complementary in-plane mirror images $(\mathrm{CL} \Gamma \mathrm{s})$ are embedded in the holes and the PEC patches of a plain checkerboard-like structure so that the metasurface with $Z_{\mathrm{s}}=Z_{0} / 2$ is congruent with the in-plane mirror image of its complementary structure. The handedness of the planar chiral metasurface is determined by the handedness of $\mathrm{R} \Gamma \mathrm{s}$ and $\mathrm{CL} \Gamma \mathrm{s}$, and by the electrical connectivity of the PEC patches. As mentioned above, electromagnetic responses of metallic checkerboard-like structures critically depend on the electrical connectivity at the interconnection points of their PEC patches. Here, we initially assume $Z_{\mathrm{s}}=Z_{\mathrm{s}}^{\text {(high) }} \gg Z_{0} / 2$. In other words, the PEC patches are virtually disconnected.

To evaluate the asymmetric transmission of the metasurface, we define the total-transmission-intensity difference $\Delta T_{\mathrm{L}}[8]$ for the LCP waves by

$$
\Delta T_{\mathrm{L}}:=\left(\vec{T}_{\mathrm{LL}}+\vec{T}_{\mathrm{RL}}\right)-\left(\bar{T}_{\mathrm{LL}}+\bar{T}_{\mathrm{RL}}\right)=\vec{T}_{\mathrm{RL}}-\bar{T}_{\mathrm{RL}},
$$

where power transmittance $T_{i j}:=\left|\tilde{t}_{i j}\right|^{2}$ and the symbol $\rightarrow$ $(\leftarrow)$ indicate normal incidence from $z>0(z<0)$. Here, we assume that scattering into diffraction modes is negligible. In other words, the periodicity of the metasurface is smaller than the incident wavelength. Note that $\vec{T}_{\mathrm{LL}}=\bar{T}_{\mathrm{LL}}$ is satisfied owing to the reciprocity. From the mirror symmetry with respect to the $z=0$ plane, we can show $\Delta T_{\mathrm{R}}=-\Delta T_{\mathrm{L}}$ for the normal incidence of the RCP waves. Thus, we will omit the subscript as $\Delta T:=\Delta T_{\mathrm{L}}$ for simplicity below. The sign of $\Delta T$ is determined by the handedness of the planar chiral metasurface observed from $z>0$. We note that $\Delta T$ can also be written as $\Delta T=\vec{T}_{\mathrm{RL}}-\vec{T}_{\mathrm{LR}}$ because we have $\bar{T}_{\mathrm{RL}}=\vec{T}_{\mathrm{LR}}$ from the reciprocity. Thus, the asymmetric transmission for circularly polarized waves can 


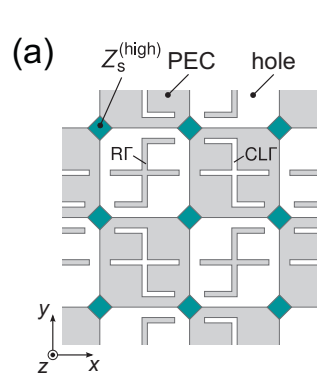

high-impedance structure

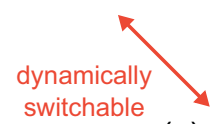

(c)

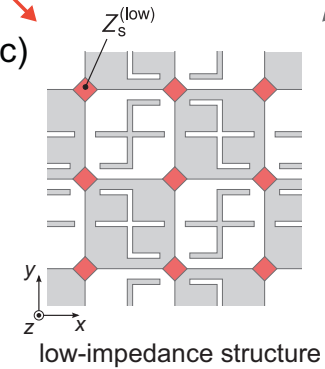

(a)

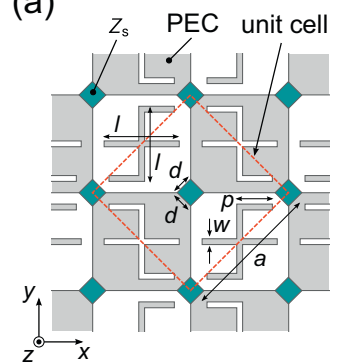

(b)

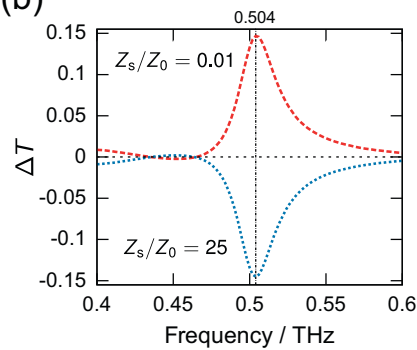

(c)

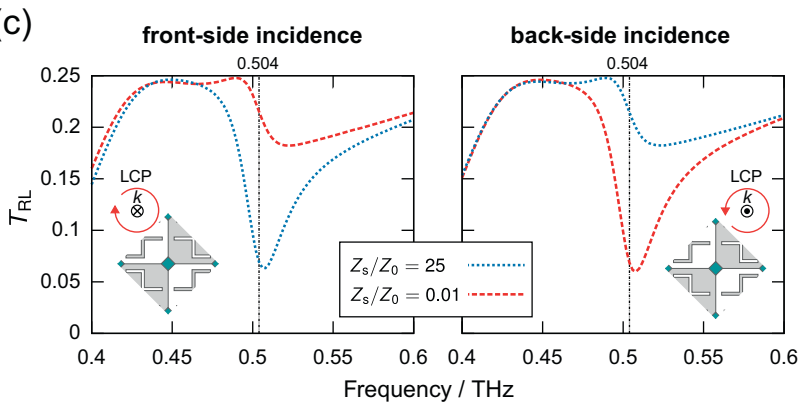

Figure 3. (a) Unit cell of the metasurface in the numerical simulation. The geometric parameters are as follows: $a=300 \mu \mathrm{m}$, $d=30 \mu \mathrm{m}, l=180 \mu \mathrm{m}, p=80 \mu \mathrm{m}$, and $w=10 \mu \mathrm{m}$; (b) calculated $\Delta T$ spectra for $Z_{\mathrm{S}} / Z_{0}=25$ and 0.01 ; (c) calculated spectra of $\vec{T}_{\mathrm{RL}}$ and $\bar{T}_{\mathrm{RL}}$ for $Z_{\mathrm{S}} / Z_{0}=25$ and 0.01 .

$\vec{T}_{\mathrm{RL}}^{(\text {low })}=\bar{T}_{\mathrm{RL}}^{(\mathrm{c})}$ and $\bar{T}_{\mathrm{RL}}^{\text {(low })}=\vec{T}_{\mathrm{RL}}^{(\mathrm{c})}$, where the superscript "(low)" represents the low-impedance structure. Finally, we obtain the following equations:

$$
\Delta T^{(\text {low })}=-\Delta T^{(\mathrm{c})}=-\Delta T^{(\text {high })},
$$

where equation (5) is used. This equation indicates that the sign of $\Delta T$ is inverted just by switching the sheet impedance at the corners. Therefore, the handedness of the planar chiral metasurface is dynamically switchable by using variable impedance sheets. Note that we have $\Delta T^{(\text {low })}=-\Delta T^{(\text {high) }}=0$ in the limit of $Z_{\mathrm{s}}^{(\text {high })}=\infty$ and $Z_{\mathrm{s}}^{(\text {low })}=0$, where the metasurface is lossless. This is because loss is essential for the asymmetric transmission in planar chiral structures [8]. We also note that $\Delta T^{(\mathrm{c})}=-\Delta T^{(\text {high) }}=-\Delta T^{(\text {low })}=0$ if $Z_{\mathrm{s}}^{(\text {high) }}=Z_{\mathrm{s}}^{(\text {low })}=Z_{0} / 2$.

\section{Numerical simulation}

\subsection{Planar chiral checkerboard-like metasurface in a vacuum}

In order to validate the theoretical analysis in Section 2, we numerically simulated the electromagnetic responses of the proposed metasurface using the finite element method solver (ANSYS HFSS). Figure 3a shows the unit cell of the simulated metasurface, which was placed at $z=0$ in the computational domain of a vacuum. The geometrical parameters in Figure 3a are: $a=300 \mu \mathrm{m}, d=30 \mu \mathrm{m}, l=180 \mu \mathrm{m}$, $p=80 \mu \mathrm{m}$, and $w=10 \mu \mathrm{m}$. The CL $\Gamma \mathrm{s}$ have the same parameters as the $R \Gamma \mathrm{s}$. There are sheets with variable sheet impedances $Z_{\mathrm{s}}$ at the interconnection points of the PEC patches. tion of Figure $2 b$ from the opposite $(z<0)$ side. Thus, we have interconnection points from $Z_{\mathrm{s}}^{\text {(high) }}$ to $Z_{\mathrm{s}}^{(\text {low })} \ll Z_{0} / 2$, the metasurface shown in Figure 2 a changes to the structure shown in Figure 2c. If $Z_{\mathrm{s}}^{\text {(low) }}=Z_{0}^{2} /\left(4 Z_{\mathrm{s}}^{\text {(high) }}\right)$ is satisfied, the lowimpedance structure shown in Figure $2 \mathrm{c}$ is congruent with the in-plane mirror image of the complementary structure
shown in Figure $2 \mathrm{~b}$. In other words, Figure $2 \mathrm{c}$ is the observa- 


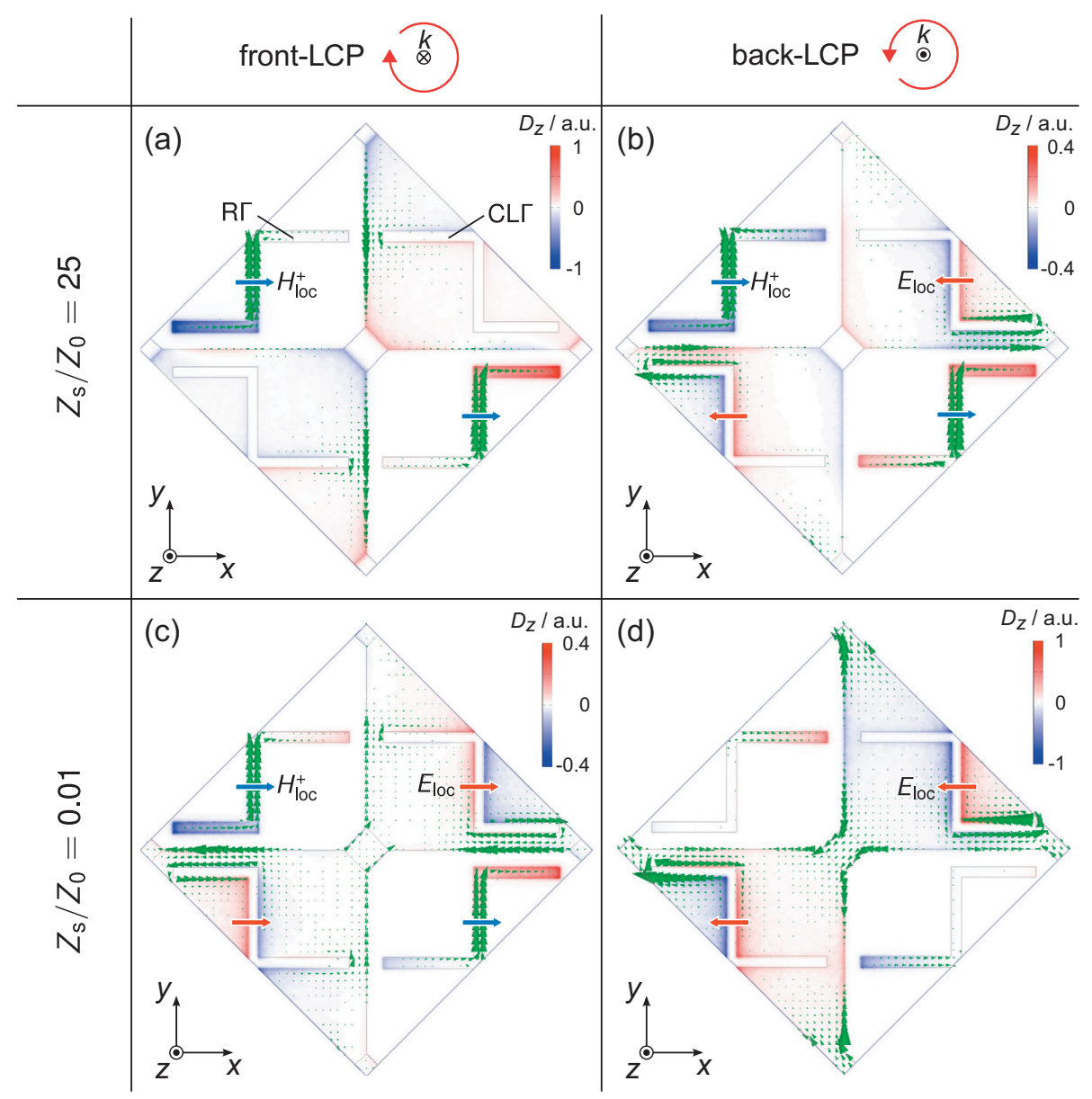

Figure 4. Distributions of the electric flux density $D_{z}$ at $z=1 \mu \mathrm{m}$ and surface current $J_{\text {surf }}$ on the metasurface for the incidence of the LCP waves from the front $(z>0)$ or back $(z<0)$ sides of the metasurface structure at $0.504 \mathrm{THz}$ : (a) $Z_{\mathrm{s}} / Z_{0}=25$ with front-side incidence; (b) $Z_{\mathrm{s}} / Z_{0}=25$ with back-side incidence; (c) $Z_{\mathrm{s}} / Z_{0}=0.01$ with front-side incidence; (d) $Z_{\mathrm{s}} / Z_{0}=0.01$ with back-side incidence. The color maps and arrows in green indicate $D_{z}$ and the surface current vector, respectively. For clarity, the phase of $D_{z}$ is shifted by $\pi / 2$ relative to $J_{\text {surf }}$.

We imposed periodic boundary conditions on the side boundaries of the computational domain to simulate an infinitely periodic system. In the simulation, the complex amplitude transmission coefficients for the normal incidence of $x$ and $y$ linearly polarized waves were calculated, and then, the transmission coefficients were transformed into those in the circular-polarization basis.

The calculated $\Delta T$ spectra for $Z_{\mathrm{s}} / Z_{0}=25$ and 0.01 are shown in Figure $3 \mathrm{~b}$. Note that $Z_{\mathrm{s}} / Z_{0}=25$ and 0.01 are complementary to each other via the impedance inversion. We observe $|\Delta T|$ is resonantly enhanced around $0.504 \mathrm{THz}$. In addition, it is clearly confirmed that the polarity of the transmission asymmetry is inverted by switching the sheet impedance $Z_{\mathrm{s}}$, which agrees well with equation (6). In other words, the handedness of the planar chiral metasurface is dynamically switchable by modulating its local sheet impedance $Z_{\mathrm{s}}$. Figure $3 \mathrm{c}$ shows the calculated spectra of $T_{\mathrm{RL}}$ for incidence on the front of the metasurface $(z>0)$ or back $(z<0)$. Because the problems of $Z_{\mathrm{S}} / Z_{0}=25$ are related to the problems of $Z_{\mathrm{s}} / Z_{0}=0.01$ with the opposite incident direction via Babinet's principle, as expected from equation (3), two types of $T_{\mathrm{RL}}$ spectra, one of which shows a dip around
$0.504 \mathrm{THz}$, are interchanged if the incident direction is reversed or $Z_{\mathrm{s}}$ is switched.

To understand the resonant character of $\Delta T$ around $0.504 \mathrm{THz}$, we simulated the distributions of the surface current $J_{\text {surf }}$ at $z=0$ and the $z$ component of the electric flux density $D_{z}$ at $z=1 \mu \mathrm{m}$, which approximately corresponds to the surface charge density on the metasurface, for the normal incidence of the LCP waves at $0.504 \mathrm{THz}$. Figure 4 shows the snapshots of the distributions at the phase when $J_{\text {surf }}$ takes its maximum value. For clarity, the phase of $D_{z}$ is shifted by $\pi / 2$ relative to $J_{\text {surf. }}$.

In the case of $Z_{\mathrm{s}} / Z_{0}=25$ with front-side incidence of the LCP waves shown in Figure 4a, a dipolar current along the $y$ direction is induced on $\mathrm{R} \Gamma \mathrm{s}$, which causes a local magnetic field $H_{\mathrm{loc}}^{+}(z=+0)=-H_{\mathrm{loc}}^{-}(z=-0)$ in the $x$ direction around the bars of $\mathrm{R} \Gamma \mathrm{s}$. Generally, such a dipolar current contributes to circular polarization conversion because its $y$-polarized emission is a superposition of the LCP and RCP waves [9]. However, the calculated $T_{\mathrm{RL}}$ spectrum shown in Figure $3 \mathrm{c}$ shows a dip around $0.504 \mathrm{THz}$. Therefore, the emission from the resonance mode in $R \Gamma \mathrm{s}$ destructively interferes with that from the charge accumulated at the corners of the 


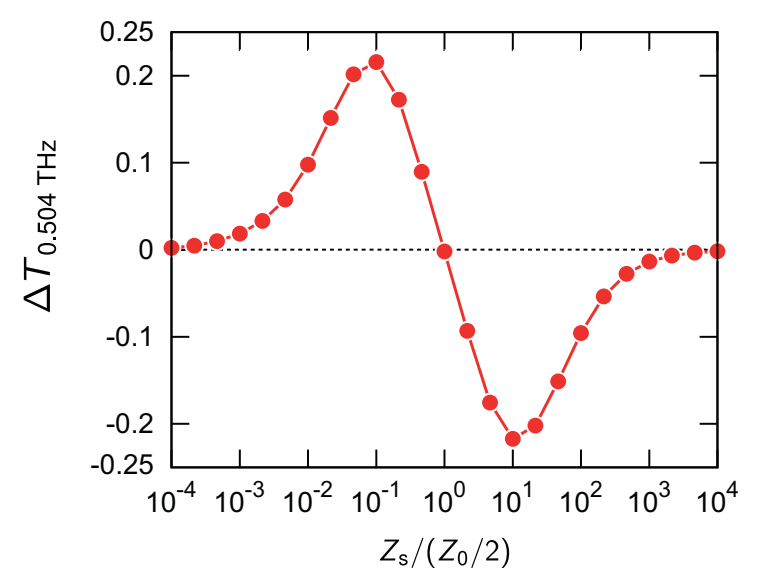

Figure 5. Calculated $Z_{\mathrm{s}}$ dependence of $\Delta T$ at $0.504 \mathrm{THz}$.

checkerboard structure (see Appendix). As mentioned above, the case of $Z_{\mathrm{s}} / Z_{0}=0.01$ with back-side incidence shown in Figure $4 \mathrm{~d}$ is the complementary problem of the case shown in Figure 4a. In Figure 4d, we confirm a loop current induced around the arms of $\mathrm{CL} \Gamma \mathrm{s}$ and charge accumulation along the slits of CLГs in the $y$ direction, which causes a local electric field $E_{\mathrm{loc}}$ in the $x$ direction in the slits of CL $\Gamma$ s. This resonance mode characterized by $E_{\text {loc }}$ is complementary to the one characterized by $H_{\text {loc }}$ in Figure 4a. We note that the polarization of the waves emitted from the complementary mode of CL $\Gamma \mathrm{s}$ is rotated by $\pi / 2$ compared to that from the $\mathrm{R} \Gamma \mathrm{s}$ due to the interchange of $\boldsymbol{E}$ and $\boldsymbol{H}$ in Babinet's principle. The dip in the $T_{\mathrm{RL}}$ spectrum can be explained in the same way as above.

On the other hand, in the cases of $Z_{\mathrm{s}} / Z_{0}=25$ with backside incidence (Figure $4 \mathrm{~b}$ ) and $Z_{\mathrm{s}} / Z_{0}=0.01$ with front-side incidence (Figure $4 \mathrm{c}$ ), both the resonance modes of $R \Gamma \mathrm{s}$ and $\mathrm{CL} \Gamma \mathrm{s}$ are excited. The phase maximizing $E_{\mathrm{loc}}$ in CLCs is delayed by approximately $\pi / 2$, relative to that maximizing $H_{\text {loc }}$ in $\mathrm{R} \Gamma \mathrm{s}$. Here, note that $D_{z}$ is depicted with a $\pi / 2$ phase shift relative to $J_{\text {surf }}$ in Figure 4 . Hence, the RCP components are dominantly emitted from $\mathrm{R} \Gamma \mathrm{s}$ and $\mathrm{CL} \Gamma \mathrm{s}$ in the $\boldsymbol{k}$ directions of Figures $4 \mathrm{~b}$ and $4 \mathrm{c}$, and the corresponding $T_{\mathrm{RL}}$ exhibits a relatively large value around $0.504 \mathrm{THz}$, as shown in Figure 3c.

From an application perspective, continuous tunability of $\Delta T$ is also a desired feature. Figure 5 shows the calculated $Z_{\mathrm{s}}$ dependence of $\Delta T$ at $0.504 \mathrm{THz}$. It is confirmed that $\Delta T$ can be continuously tuned by controlling $Z_{\mathrm{s}}$ around $Z_{0} / 2$. As predicted in Section 2, $\Delta T$ tends to zero when $Z_{\mathrm{s}} \rightarrow \infty$ or 0 due to the disappearance of lossy elements. $\Delta T$ is also nearly zero at $Z_{\mathrm{s}}=Z_{0} / 2$. The maximum value of $|\Delta T|$ is about 0.22 in the present case. There is little room for further increasing $|\Delta T|$ by optimizing the geometry of the deformed gammadions as $T_{\mathrm{RL}}$ is theoretically bound by 0.25 for electric single-layer structures [37].

\subsection{Planar chiral checkerboard-like metasurface on a dielectric substrate}

To actually materialize the proposed metasurface, there must be a dielectric substrate supporting the thin metasurface

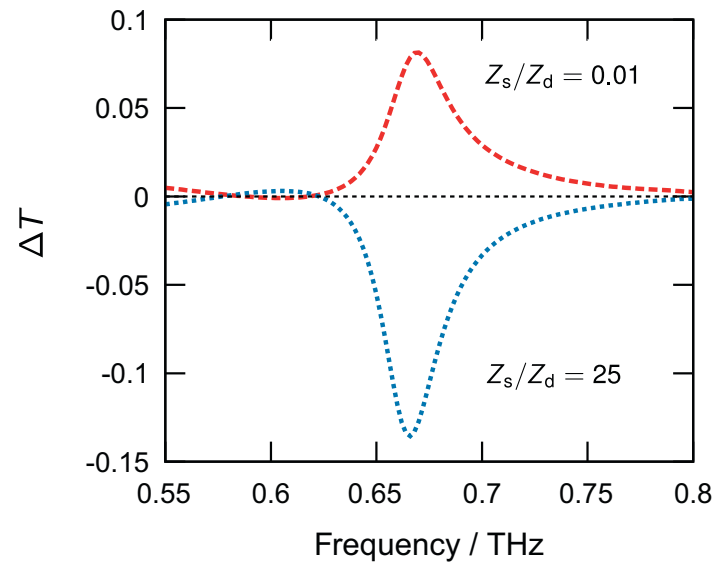

Figure 6. Calculated $\Delta T$ spectra of the planar chiral checkerboardlike metasurface on a dielectric substrate for $Z_{\mathrm{s}} / Z_{\mathrm{d}}=0.01$ and 25 .

structure. The existence of the substrate breaks the mirror symmetry with respect to $z=0$ and thereby invalidates Babinet's principle and equation (6). This difficulty can be approximately circumvented by just sandwiching the metasurface structure between the same dielectric plates [31]. In addition, it is reasonable to expect that similar responses are obtained if the metasurface is placed on a dielectric substrate with a moderate refractive index. Here, we will numerically demonstrate that sign inversion of $\Delta T$ can occur for a metasurface placed on a dielectric substrate. We simulated a similar model to Figure 3a with the computational domain in $z<0$ filled by a lossless dielectric material having a refractive index of $n=3.4$, which corresponds to that of highresistivity silicon in the terahertz regime [38]. To reflect the wavelength-shortening effect in the substrate, all the geometrical parameters were divided by $n$. Figure 6 shows the calculated $\Delta T$ spectra for $Z_{\mathrm{s}} / Z_{\mathrm{d}}=25$ and 0.01 , where $Z_{\mathrm{d}}:=Z_{0} / n$. We can confirm a sign inversion of $\Delta T$, although the spectral shapes of $|\Delta T|$ are not identical between the two cases. This is attributed to the broken mirror symmetry with respect to $z=0$ caused by the substrate.

\section{Conclusion}

In conclusion, we investigated the dynamical handedness switching in a planar chiral checkerboard-like metasurface loaded with switchable impedance sheets. We theoretically and numerically showed that the polarity of the asymmetric transmission in the proposed planar chiral metasurface can be inverted by modulating the sheet impedance at the interconnection points of the checkerboard-like structure. Such impedance switching can be experimentally implemented in the terahertz regime by exploiting, for example, photoexcitation of semiconductors $[16,17,39]$ and metal-insulator transitions of vanadium dioxide $[33,34]$. We explained the behaviors of the metasurface from the microscopic point of view, i.e., the field distributions. Note that we can also discuss them in terms of macroscopic parameters such as averaged sheet admittance tensors [40, 41], which facilitate design of metasurfaces with desired transmission and reflection 
characteristics. Finally, it should be noted that although the simulation was performed in the terahertz regime, the theory discussed in this paper is also applicable to the other frequency ranges, where metals exhibit high conductivity and, thus, Babinet's principle is valid.

Acknowledgements. This research was partially supported by JSPS KAKENHI Grant Numbers JP15J07603 and JP25790065 and by grant from the Murata Science Foundation. One of the authors (Y.U.) was supported by the JSPS Research Fellowships for Young Scientists.

\section{References}

1. Z. Li, M. Mutlu, E. Ozbay, Chiral metamaterials: from optical activity and negative refractive index to asymmetric transmission, J. Opt. 15 (2013) 023001.

2. G. Kenanakis, E.N. Economou, C.M. Soukoulis, M. Kafesaki, Controlling THz and far-IR waves with chiral and bianisotropic metamaterials, EPJ Appl. Metamat. 2 (2015) 15.

3. L.R. Arnaut, Chirality in multi-dimensional space with application to electromagnetic characterisation of multi-dimensional chiral and semi-chiral media, J. Electromagn. Waves Appl. 11 (1997) 1459-1482.

4. M. Kuwata-Gonokami, N. Saito, Y. Ino, M. Kauranen, K. Jefimovs, T. Vallius, J. Turunen, Y. Svirko, Giant optical activity in quasi-two-dimensional planar nanostructures, Phys. Rev. Lett. 95 (2005) 227401.

5. L. Hecht, L.D. Barron, Rayleigh and Raman optical activity from chiral surfaces, Chem. Phys. Lett. 225 (1994) 525-530.

6. A. Papakostas, A. Potts, D.M. Bagnall, S.L. Prosvirnin, H.J. Coles, N.I. Zheludev, Optical manifestations of planar chirality, Phys. Rev. Lett. 90 (2003) 107404

7. S.L. Prosvirnin, N.I. Zheludev, Polarization effects in the diffraction of light by a planar chiral structure, Phys. Rev. E 71 (2005) 037603.

8. V.A. Fedotov, P.L. Mladyonov, S.L. Prosvirnin, A.V. Rogacheva, Y. Chen, N.I. Zheludev, Asymmetric propagation of electromagnetic waves through a planar chiral structure, Phys. Rev. Lett. 97 (2006) 167401.

9. E. Plum, V.A. Fedotov, N.I. Zheludev, Planar metamaterial with transmission and reflection that depend on the direction of incidence, Appl. Phys. Lett. 94 (2009) 131901.

10. R. Singh, E. Plum, C. Menzel, C. Rockstuhl, A.K. Azad, R.A. Cheville, F. Lederer, W. Zhang, N.I. Zheludev, Terahertz metamaterial with asymmetric transmission, Phys. Rev. B 80 (2009) 153104.

11. A.S. Schwanecke, V.A. Fedotov, V.V. Khardikov, S.L. Prosvirnin, Y. Chen, N.I. Zheludev, Nanostructured metal film with asymmetric optical transmission, Nano Lett. 8 (2008) 2940-2943.

12. C. Menzel, C. Helgert, C. Rockstuhl, E.B. Kley, A. Tünnermann, T. Pertsch, F. Lederer, Asymmetric transmission of linearly polarized light at optical metamaterials, Phys. Rev. Lett. 104 (2010) 253902.

13. N.I. Zheludev, Y.S. Kivshar, From metamaterials to metadevices, Nat. Mater. 11 (2012) 917-924.

14. A.M. Shaltout, A.V. Kildishev, V.M. Shalaev, Evolution of photonic metasurfaces: from static to dynamic, J. Opt. Soc. Am. B 33 (2016) 501-510.
15. N. Kanda, K. Konishi, M. Kuwata-Gonokami, Light-induced terahertz optical activity, Opt. Lett. 34 (2009) 3000-3002.

16. J. Zhou, D.R. Chowdhury, R. Zhao, A.K. Azad, H.-T. Chen, C.M. Soukoulis, A.J. Taylor, J.F. O'Hara, Terahertz chiral metamaterials with giant and dynamically tunable optical activity, Phys. Rev. B 86 (2012) 035448.

17. S. Zhang, J. Zhou, Y.-S. Park, J. Rho, R. Singh, S. Nam, A.K. Azad, H.-T. Chen, X. Yin, A.J. Taylor, X. Zhang, Photoinduced handedness switching in terahertz chiral metamolecules, Nat. Commun. 3 (2012) 942.

18. N. Kanda, K. Konishi, M. Kuwata-Gonokami, Allphotoinduced terahertz optical activity, Opt. Lett. 39 (2014) 3274-3277.

19. G. Kenanakis, R. Zhao, N. Katsarakis, M. Kafesaki, C.M. Soukoulis, E.N. Economou, Optically controllable THz chiral metamaterials, Opt. Express 22 (2014) 12149-12159.

20. T. Kan, A. Isozaki, N. Kanda, N. Nemoto, K. Konishi, M. Kuwata-Gonokami, K. Matsumoto, I. Shimoyama, Spiral metamaterial for active tuning of optical activity, Appl. Phys. Lett. 102 (2013) 221906.

21. T. Kan, A. Isozaki, N. Kanda, N. Nemoto, K. Konishi, H. Takahashi, M. Kuwata-Gonokami, K. Matsumoto, I. Shimoyama, Enantiomeric switching of chiral metamaterial for terahertz polarization modulation employing vertically deformable MEMS spirals, Nat. Commun. 6 (2015) 8422.

22. X. Yin, M. Schäferling, A.-K.U. Michel, A. Tittl, M. Wuttig, T. Taubner, H. Giessen, Active chiral plasmonics, Nano Lett. 15 (2015) 4255-4260.

23. R.C. Compton, J.C. Macfarlane, L.B. Whitbourn, M.M. Blanco, R.C. McPhedran, Babinet's principle applied to ideal beam-splitters for submillimetre waves, Opt. Acta 31 (1984) 515-524.

24. K. Kempa, Percolation effects in the checkerboard Babinet series of metamaterial structures, Phys. Status Solidi Rapid Res. Lett. 4 (2010) 218-220.

25. J.D. Edmunds, A.P. Hibbins, J.R. Sambles, I.J. Youngs, Resonantly inverted microwave transmissivity threshold of metal grids, New J. Phys. 12 (2010) 063007.

26. S.A. Ramakrishna, P. Mandal, K. Jeyadheepan, N. Shukla, S. Chakrabarti, M. Kadic, S. Enoch, S. Guenneau, Plasmonic interaction of visible light with gold nanoscale checkerboards, Phys. Rev. B 84 (2011) 245424.

27. K. Takano, F. Miyamaru, K. Akiyama, H. Miyazaki, M.W. Takeda, Y. Abe, Y. Tokuda, H. Ito, M. Hangyo, Crossover from capacitive to inductive electromagnetic responses in near self-complementary metallic checkerboard patterns, Opt. Express 22 (2014) 24787-24795.

28. D. González-Ovejero, E. Martini, S. Maci, Surface waves supported by metasurfaces with self-complementary geometries, IEEE Trans. Antennas Propag. 63 (2015) 250-260.

29. B. Tremain, C.J. Durrant, I.E. Carter, A.P. Hibbins, J.R. Sambles, The effect of rotational disorder on the microwave transmission of checkerboard metal square arrays, Sci. Rep. 5 (2015) 16608.

30. Y. Nakata, Y. Urade, T. Nakanishi, M. Kitano, Plane-wave scattering by self-complementary metasurfaces in terms of electromagnetic duality and Babinet's principle, Phys. Rev. B 88 (2013) 205138.

31. Y. Urade, Y. Nakata, T. Nakanishi, M. Kitano, Frequencyindependent response of self-complementary checkerboard screens, Phys. Rev. Lett. 114 (2015) 237401. 
32. Y. Urade, Y. Nakata, T. Nakanishi, M. Kitano, Broadband and energy-concentrating terahertz coherent perfect absorber based on a self-complementary metasurface, Opt. Lett. 41 (2016) 4472-4475.

33. Y. Urade, Y. Nakata, K. Okimura, T. Nakanishi, F. Miyamaru, M.W. Takeda, M. Kitano, Dynamically Babinet-invertible metasurface: a capacitive-inductive reconfigurable filter for terahertz waves using vanadium-dioxide metal-insulator transition, Opt. Express 24 (2016) 4405-4410.

34. Y. Nakata, Y. Urade, K. Okimura, T. Nakanishi, F. Miyamaru, M.W. Takeda, M. Kitano, Anisotropic Babinet-invertible metasurfaces to realize transmission-reflection switching for orthogonal polarizations of light, Phys. Rev. Applied 6 (2016) 044022.

35. R. Zhao, L. Zhang, J. Zhou, Th Koschny, C.M. Soukoulis, Conjugated gammadion chiral metamaterial with uniaxial optical activity and negative refractive index, Phys. Rev. B 83 (2011) 035105.

36. B. Kang, K. Takano, M. Hangyo, Asymmetric transmission of planar chiral $\mathrm{THz}$ metamaterials for circularly polarized light, in 38th International Conference on Infrared, Millimeter, and Terahertz Waves (IRMMW-THz 2013), Mainz, Germany, 2013.

37. X. Ding, F. Monticone, K. Zhang, L. Zhang, D. Gao, S.N. Burokur, A. de Lustrac, Q. Wu, C.-W. Qiu, A. Alù, Ultrathin Pancharatnam-Berry metasurface with maximal cross-polarization efficiency, Adv. Mater. 27 (2015) 1195-1200.

38. D. Grischkowsky, S. Keiding, M. van Exter, Ch Fattinger, Far-infrared time-domain spectroscopy with terahertz beams of dielectrics and semiconductors, J. Opt. Soc. Am. B 7 (1990) 2006.

39. N.-H. Shen, M. Massaouti, M. Gokkavas, J.-M. Manceau, E. Ozbay, M. Kafesaki, T. Koschny, S. Tzortzakis, C.M. Soukoulis, Optically implemented broadband blueshift switch in the terahertz regime, Phys. Rev. Lett. 106 (2011) 037403.

40. P. Tassin, T. Koschny, C.M. Soukoulis, Effective material parameter retrieval for thin sheets: theory and application to graphene, thin silver films, and single-layer metamaterials, Physica B 407 (2012) 4062-4065.

41. C. Pfeiffer, A. Grbic, Bianisotropic metasurfaces for optimal polarization control: analysis and synthesis, Phys. Rev. Applied 2 (2014) 044011.

42. A.E. Miroshnichenko, S. Flach, Y.S. Kivshar, Fano resonances in nanoscale structures, Rev. Mod. Phys. 82 (2010) 2257-2298.

\section{Appendix}

\section{Origin of the dip in the cross-polarized transmission spectra}

To understand the origin of the dip around $0.504 \mathrm{THz}$ in the $T_{\mathrm{RL}}$ spectra, we calculated $T_{\mathrm{RL}}$ spectra of the metasurface without gammadions of one handedness. Figure 7 shows the $\vec{T}_{\mathrm{RL}}$ spectra of the metasurface with and without $\mathrm{R} \Gamma \mathrm{s}$ for $Z_{\mathrm{s}} / Z_{0}=25$. While the spectrum is relatively flat in the case without $\mathrm{R} \Gamma \mathrm{s}$, the case with $\mathrm{R} \Gamma \mathrm{s}$ shows an acute spectral change around $0.5 \mathrm{THz}$. In addition, we can confirm the enhancement of conversion around $0.46 \mathrm{THz}$ when $\mathrm{R} \Gamma \mathrm{s}$ are added to the structure. These observations can be understood as in the case of Fano resonance [42] by considering constructive and destructive interference between the resonance of $\mathrm{R} \Gamma \mathrm{s}$ and the broader background spectrum of the checkerboard metasurface without $\mathrm{R} \Gamma \mathrm{s}$, which is caused by the charge accumulated at the corners of the checkerboard structure.

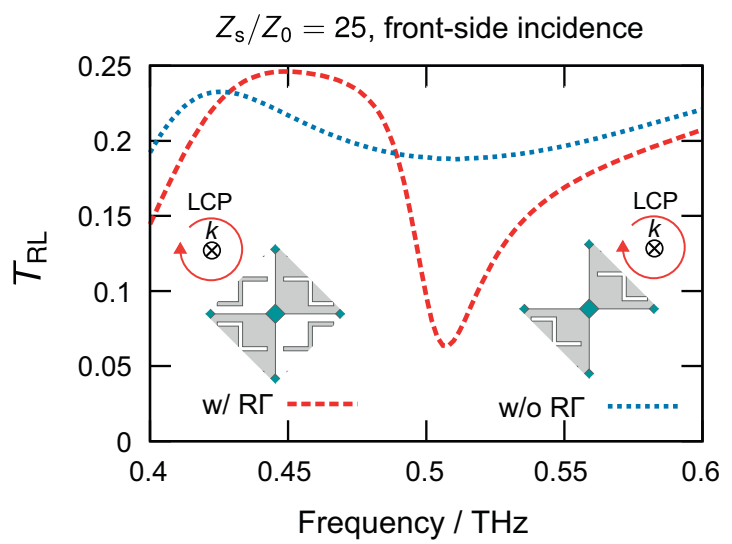

Figure 7. Calculated $\vec{T}_{\mathrm{RL}}$ spectra of the metasurface with and without $\mathrm{R} \Gamma \mathrm{s}$ for $Z_{\mathrm{s}} / Z_{0}=25$.

Cite this article as: Urade Y, Nakata Y, Nakanishi T \& Kitano M: Theoretical study on dynamical planar-chirality switching in checkerboard-like metasurfaces. EPJ Appl. Metamat. 2017, 4, 2. 